\title{
Higher serum 25(OH)D concentrations are associated with improved FEV1 and FVC in adolescence
}

\author{
Claudia Flexeder ${ }^{1}$, Elisabeth Thiering ${ }^{1,2}$, Sibylle Koletzko ${ }^{2}$, Dietrich Berdel ${ }^{3}$, \\ Irina Lehmann ${ }^{4}$, Andrea von Berg ${ }^{3}$, Barbara Hoffmann ${ }^{5}$, Carl-Peter Bauer ${ }^{6}$, \\ Joachim Heinrich ${ }^{1,7,8}$ and Holger Schulz $z^{1,7}$
}

\begin{abstract}
Affiliations: ${ }^{1}$ Helmholtz Zentrum München, German Research Center for Environmental Health, Institute of Epidemiology I, Neuherberg, Germany. ${ }^{2}$ Ludwig Maximilians University of Munich, Dr. von Hauner Children's Hospital, Munich, Germany. ${ }^{3}$ Marien-Hospital Wesel, Research Institute, Department of Pediatrics, Wesel, Germany. ${ }^{4}$ Helmholtz Centre for Environmental Research - UFZ, Department of Environmental Immunology, Leipzig, Germany. ${ }^{5}$ Heinrich Heine University of Düsseldorf, IUF Leibniz Research Institute for Environmental Medicine and Medical Faculty, Düsseldorf, Germany. ${ }^{6}$ Technical University of Munich, Department of Pediatrics, Munich, Germany. ${ }^{7}$ Comprehensive Pneumology Center Munich (CPC-M). Member of the German Center for Lung Research, Munich, Germany. ${ }^{8}$ Ludwig Maximilians University Munich, University Hospital Munich, Institute and Outpatient Clinic for Occupational, Social and Environmental Medicine, Munich, Germany.
\end{abstract}

Correspondence: Holger Schulz, Institute of Epidemiology I, Helmholtz Zentrum München, German Research Center for Environmental Health, Ingolstädter Landstraße 1, 85764 Neuherberg, Germany.

E-mail: schulzahelmholtz-muenchen.de

@ERSpublications

Serum $25(\mathrm{OH})$ D concentrations are positively associated with volume-related lung function parameters in adolescence http://ow.ly/6WmC309dLrS

Cite this article as: Flexeder C, Thiering E, Koletzko S, et al. Higher serum 25(OH)D concentrations are associated with improved FEV1 and FVC in adolescence. Eur Respir J 2017; 49: 1601804 [https://doi.org/ 10.1183/13993003.01804-2016].

ABSTRACT Vitamin D plays a role in the development of the immune system and the lung, as well as in airway remodelling. Therefore, this study investigated the association between serum 25 -hydroxyvitamin D $(25(\mathrm{OH}) \mathrm{D})$ concentrations and spirometric lung function parameters at age 15 years.

In the German birth cohorts GINIplus and LISAplus, lung function testing by spirometry and $25(\mathrm{OH}) \mathrm{D}$ measurements were performed during the 15-year follow-up examinations. Valid lung function measurements pre- and/or post-bronchodilation and serum $25(\mathrm{OH}) \mathrm{D}$ concentrations, which were adjusted for the date of blood sampling to account for seasonal variability, were available for 2607 adolescents. Associations between $25(\mathrm{OH}) \mathrm{D}$ concentrations and spirometric parameters were analysed using generalised additive models adjusted for confounding factors.

Serum 25(OH)D concentrations were significantly associated with forced vital capacity (FVC), forced expiratory volume in $1 \mathrm{~s}$ (FEV1) and $\mathrm{FEV}_{1} / \mathrm{FVC}$ measured before bronchodilation after adjustment for potential confounders: FEV1 increased by $10 \mathrm{~mL}$ (95\% CI 2-17), FVC by $20 \mathrm{~mL}$ (95\% CI 12-28) and FEV1/ FVC decreased by $0.177 \%$ (95\% CI -0.286 to -0.067$)$ per $10 \mathrm{nmol} \cdot \mathrm{L}^{-1}$ increase in $25(\mathrm{OH}) \mathrm{D}$ concentrations. Flow rates (forced expiratory flow rates at 25, 50 and 75\% of exhaled FVC (FEF25, FEF50, FEF75) and mean flow rate between 25 and $75 \%$ of FVC (FEF25-75)) were not associated with vitamin D. Similar associations were observed for lung function parameters measured after bronchodilation.

Vitamin D concentrations are positively associated with volume-related lung function parameters preand post-bronchodilation, suggesting structural changes in peripheral airways.

Received: Sept 122016 | Accepted after revision: Jan 112017

Conflict of interest: Disclosures can be found alongside this article at erj.ersjournals.com

Copyright @ERS 2017 


\section{Introduction}

Humans obtain $10 \%$ of their vitamin D from food intake and $90 \%$ from endogenous synthesis after sunlight exposure [1]. Vitamin $\mathrm{D}_{3}$ (cholecalciferol) is metabolised in the liver to 25-hydroxyvitamin $\mathrm{D}_{3}$, which is then converted in the kidney to its active metabolite 1,25-dihydroxyvitamin $\mathrm{D}_{3}$. $25-$ Hydroxyvitamin $\mathrm{D}(25(\mathrm{OH}) \mathrm{D})$ is the main circulating vitamin $\mathrm{D}$ metabolite and can best represent vitamin D status in an individual [1-3]. The serum half-life of $25(\mathrm{OH}) \mathrm{D}$ is approximately 3 weeks [3]. As vitamin $\mathrm{D}$ production depends on the action of ultraviolet radiation, it shows a seasonal pattern $[1,3]$.

Besides its role in calcium and phosphate homeostasis, vitamin D is assumed to be related to allergic diseases [4]. Several studies have investigated the effect of vitamin D concentrations on the prevalence of asthma and other allergic diseases, but the findings are inconsistent [1,4-6]. Some studies found a protective effect of vitamin $\mathrm{D}$ on the onset of allergic diseases and asthma, whereas other studies showed an adverse effect or no relationship.

The relationship between vitamin $\mathrm{D}$ and allergies might be explained by the immunomodulatory properties of vitamin D [1, 4]. For instance, the production of anti-inflammatory cytokines (such as IL-10) is increased, whereas the production of pro-inflammatory cytokines (such as IL-12) is reduced. Vitamin D can also inhibit the proliferation and growth of smooth muscle cells of the airways and thus play a role in airway remodelling in asthmatics [4, 7].

Although asthma is related to lower lung function, only a few studies have also investigated the association between $25(\mathrm{OH}) \mathrm{D}$ concentrations and lung function in children $[8,9]$, adolescents $[10,11]$ and adults $[10$, 12-16]. However, the results were inconclusive, as some studies found a positive relationship with lung function $[8,10]$, but others found no association $[9,11]$. Furthermore, most studies only considered lung function measurements pre-bronchodilation. Consequently, the aim of this study was to analyse the association between serum $25(\mathrm{OH}) \mathrm{D}$ concentrations and spirometric lung function parameters, including forced expiratory flow rates, measured pre- and post-bronchodilation, at 15 years of age in two German birth cohorts.

\section{Materials and methods \\ Study population}

Data from two German birth cohorts were combined for this cross-sectional analysis. The GINIplus study (German Infant study on the influence of Nutrition Intervention PLUS Air pollution and Genetics on Allergy development) is an ongoing population-based birth cohort study. A total of 5991 Caucasian neonates were recruited between September 1995 and July 1998 in the German cities of Munich and Wesel. The GINIplus study comprised an intervention arm $(\mathrm{n}=2252)$ and an observation $(\mathrm{n}=3739)$ arm. Only children with at least one atopic parent or sibling were allocated to the intervention group, which prospectively investigated the effect of different types of hydrolysed formula on allergy development. In the prospective population-based birth cohort LISAplus (Influence of Life-style related factors on the development of the Immune System and Allergies in East and West Germany PLUS the influence of traffic emissions and genetics), 3097 healthy term born neonates were recruited in the German cities of Munich, Wesel, Leipzig and Bad Honnef between December 1997 and January 1999. Three of the originally recruited 3097 subjects withdrew their consent to participate. Details of the study design and recruitment have been described previously [17-20].

Support statement: The GINIplus study was mainly supported for the first 3 years by the Federal Ministry for Education, Science, Research and Technology (interventional arm) and Helmholtz Zentrum Munich (former GSF) (observational arm). The 4-year, 6-year and 10-year follow-up examinations of the GINIplus study were covered by the respective budgets of the five study centres (Helmholtz Zentrum Munich (former GSF), Research Institute at Marien-Hospital Wesel, LMU Munich, TU Munich, and from 6 years onwards also from IUF - Leibniz Research-Institute for Environmental Medicine at the University of Düsseldorf) and a grant from the Federal Ministry for Environment (IUF Düsseldorf, FKZ 20462296). The 15 year follow-up examination of the GINIplus study was partially supported by the Commission of the European Communities, the 7th Framework Programme, MeDALL project, as well as by the companies Mead Johnson and Nestlé. The LISAplus studies were mainly supported for the first 2 years by the Federal Ministry for Education, Science, Research and Technology, the Helmholtz Centre for Environmental Research - UFZ in Leipzig, and the Helmholtz Zentrum Munich (former GSF). The 4-year, 6-year, 10-year and 15-year follow-up examinations were covered from the respective budgets of the four study centres: Helmholtz Zentrum Munich, Research Institute at Marien-Hospital Wesel, and UFZ Leipzig, and also IUF - Leibniz Research Institute for Environmental Medicine at the University of Düsseldorf at age 6 and 10 years. The 15-year follow-up examination of the LISAplus study was also supported by the Commission of the European Communities, the 7th Framework Programme, and the MeDALL project. This work was supported by the Comprehensive Pneumology Center Munich (CPC-M) as a member of the German Center for Lung Research. Funding information for this article has been deposited with the Crossref Funder Registry. 
At the 15 year follow-up, 3198 adolescents from the GINIplus study and 1740 adolescents from the LISAplus study participated. Of these 2018 in GINIplus and 1104 in LISAplus underwent physical examinations at follow-up. The analyses in this study are based on a subsample of 2607 adolescents (1729 from GINIplus and 878 from LISAplus) who had valid lung function measurements pre- and/or post-bronchodilation and who provided vitamin D measurements.

Both studies were approved by the respective local ethics committees, and written informed consent was obtained from all participating families.

\section{Lung function testing by spirometry}

Spirometry before bronchodilation was performed in line with American Thoracic Society/European Respiratory Society (ATS/ERS) recommendations [21] during the physical examination at the 15 year follow-ups. A pneumotachograph-type spirometer (EasyOne Worldspirometer, ndd, Zurich, Switzerland) was used to obtain flow-volume curves. The participants performed at least three, but not more than eight, trials per test under the guidance of specifically trained examiners. All tests were visually inspected according to the ATS/ERS acceptability criteria [21], resulting in 1822 subjects from GINIplus and 935 subjects from LISAplus with valid lung function measurements pre-bronchodilation.

After completion of baseline spirometry, subjects inhaled salbutamol for the bronchodilator response according to ATS/ERS recommendations [21]. Spirometry was performed $15 \mathrm{~min}$ after salbutamol inhalation with 1732 and 915 subjects from GINIplus and LISAplus, respectively, meeting the ATS/ERS acceptability criteria [21].

Spirometric indices were taken from the manoeuvre with the largest sum of forced expiratory volume in $1 \mathrm{~s}$ (FEV1) and forced vital capacity (FVC). Further parameters evaluated were FEV1/FVC, peak expiratory flow (PEF), forced expiratory flow rates at 25, 50 and 75\% of exhaled FVC (FEF25, FEF50 and FEF75) and the mean flow rate between 25 and $75 \%$ of $\mathrm{FVC}(\mathrm{FEF} 25-75)$. A positive bronchodilation response was defined as an increase of more than $12 \%$ and more than $200 \mathrm{~mL}$ in FEV1 and/or FVC measured post-bronchodilation compared to pre-bronchodilation measurements, according to ATS/ERS standards [22]. Standardised $z$-scores were calculated based on the reference equations for spirometry from the Global Lung function Initiative (GLI, http://www.ers-education.org/guidelines/global-lung-function-initiative.aspx) to allow international comparisons [23].

\section{Blood sampling and serum 25(OH)D concentrations}

Blood samples were collected during the physical examination of the 15 year follow-ups from 2929 adolescents (1903 and 1026 from GINIplus and LISAplus, respectively). Blood samples were centrifuged after collection and stored frozen at $-80^{\circ} \mathrm{C}$ until assayed for vitamin $\mathrm{D}$.

Total $25(\mathrm{OH}) \mathrm{D}$ concentrations in serum were measured by Roche's vitamin D total test on the fully automated Modular system (E170, Roche Diagnostics, Mannheim, Germany). The specificity was reported by the manufacturer as $25(\mathrm{OH}) \mathrm{D}_{2}=92 \%, 25(\mathrm{OH}) \mathrm{D}_{3}=100 \%, 1,25(\mathrm{OH})_{2} \mathrm{D}_{2}=$ not detectable, $1,25(\mathrm{OH})_{2} \mathrm{D}_{3}=$ not detectable and $24,25(\mathrm{OH})_{2} \mathrm{D}_{3}=149 \%$, and the lower limit of detection was $3 \mathrm{ng} \cdot \mathrm{mL}^{-1}$. The intra-assay coefficient of variation was $2.2-6.8 \%$ for sera with concentrations between 8.35 and $69.6 \mathrm{ng} \cdot \mathrm{mL}^{-1}$ and the inter-assay coefficient of variation as provided by the manufacturer was $3.4-13.1 \%$ for concentrations between 8.35 and $69.6 \mathrm{ng} \cdot \mathrm{mL}^{-1}$.

\section{Statistical analyses}

All analyses were performed using the statistical software R, version 3.1.3 (http://www.R-project.org) [24]. To account for seasonal variability, serum $25(\mathrm{OH}) \mathrm{D}$ concentrations were adjusted for the date of blood sampling by fitting a generalised additive model (figure 1) which allows the modelling of a nonlinear association between the dependent and independent variable [25, 26]. Residuals were then added to the overall mean of the vitamin D concentrations. This resulted in season-adjusted vitamin D concentrations with an interpretable range of values [27]. Generalised additive models were also used to analyse the linear association between season-adjusted serum 25(OH)D concentrations and spirometric lung function parameters. By applying these models, potential nonlinear effects of continuous confounders on the outcome variable were taken into account [25].

Two models with different adjustment were fitted for all spirometric outcome variables (separately preand post-bronchodilation). Model 1 (minimal) was adjusted for sex, age, height and weight at lung function measurement and a combination of study (GINIplus and LISAplus) and study region (Munich, Wesel, Leipzig and Bad Honnef) and Model 2 (main) was adjusted for all variables included in Model 1 and additionally for parental education, birthweight, maternal smoking during pregnancy, breastfeeding, 


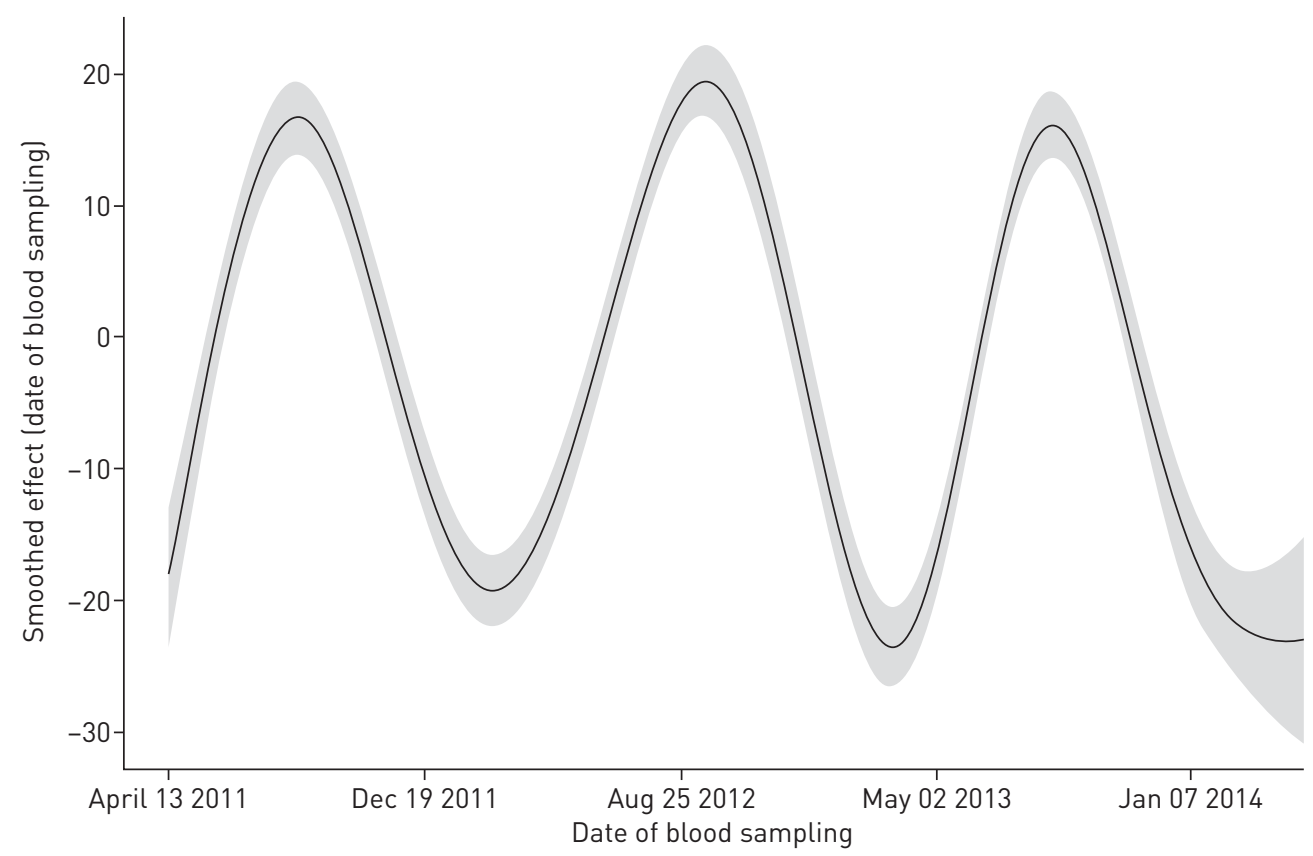

FIGURE 1 Plot showing the seasonal variability of serum 25(OH)D concentrations by fitting a generalised additive model for the smoothed association between the date of blood sampling and serum 25(OH)D concentrations.

parental atopy, exposure to tobacco smoke at home up to age 4 years, time spent in front of a television (TV)/personal computer (PC) and current asthma and/or a positive bronchodilation response.

Three categories, based on the highest number of years either mother or father attended school, were built to define parental education: low for less than 10 years, medium for 10 years, and high for more than 10 years. Parental atopy was defined as either mother or father reported having physician-diagnosed asthma, hay fever, allergic rhinitis, allergic conjunctivitis or atopic eczema. Time spent in front of a TV/PC, which was used as a surrogate for time spent inside, was defined as low if the adolescent spent not more than $1 \mathrm{~h}$ during summer and $2 \mathrm{~h}$ during winter in front of a TV or PC, high for more than $1 \mathrm{~h}$ during summer and more than $2 \mathrm{~h}$ during winter, and otherwise as medium. Current asthma was defined based on questions related to physician-diagnosed asthma ever, current wheezing at 15 years of age, and asthma medication at 15 years. An adolescent was defined as asthmatic if at least two of the three questions were answered yes.

Sensitivity analyses were conducted where asthmatics and adolescents with a positive bronchodilation response were excluded.

The results for the association between vitamin $\mathrm{D}$ and lung function indices are presented as regression coefficients $(\beta)$ per $10 \mathrm{nmol} \cdot \mathrm{L}^{-1}$ increase in season-adjusted $25(\mathrm{OH}) \mathrm{D}$ concentrations with corresponding 95\% confidence interval (CI). Differences between males and females were tested using t-test or Pearson's chi-squared test.

\section{Results}

Table 1 shows the characteristics of the total study population as well as for males and females separately. The mean of the season-adjusted $25(\mathrm{OH}) \mathrm{D}$ concentrations in the total study population was $66.3 \mathrm{nmol} \cdot \mathrm{L}^{-1}$. Mean concentrations did not differ significantly between males $\left(65.7 \mathrm{nmol} \cdot \mathrm{L}^{-1}\right)$ and females $\left(66.9 \mathrm{nmol} \cdot \mathrm{L}^{-1}\right)$. Current asthma and/or a positive bronchodilation response were diagnosed in $10.6 \%$ of the study participants, and males tended to have a higher prevalence than females ( $12.0 \%$ versus $9.2 \%)$.

Mean and standard deviation of the spirometric lung function parameters measured pre- and postbronchodilation as well as for the corresponding GLI $z$-scores are presented in table 2. Females had a higher mean FEV1/FVC ratio, pre- and post-bronchodilation, compared to males. Regarding the other lung function parameters, males had higher lung function values than females. For the sex-stratified mean GLI $z$-scores it was the other way round. Overall, the means of the GLI $z$-scores pre-bronchodilation were slightly negative.

The results for the association between season-adjusted $25(\mathrm{OH}) \mathrm{D}$ concentrations and spirometric lung function measurements pre-bronchodilation are summarised in table 3. In the minimal model, FEV1 and FVC increased by $13 \mathrm{~mL}(\beta=0.013,95 \%$ CI $0.007-0.020)$ and $24 \mathrm{~mL}(\beta=0.024,95 \%$ CI $0.017-0.031)$, respectively, per $10 \mathrm{nmol} \cdot \mathrm{L}^{-1}$ increase in season-adjusted $25(\mathrm{OH}) \mathrm{D}$ concentrations. These associations also 
TABLE 1 Study characteristics of participants at the 15 year follow-up, with valid lung function and vitamin D measurements

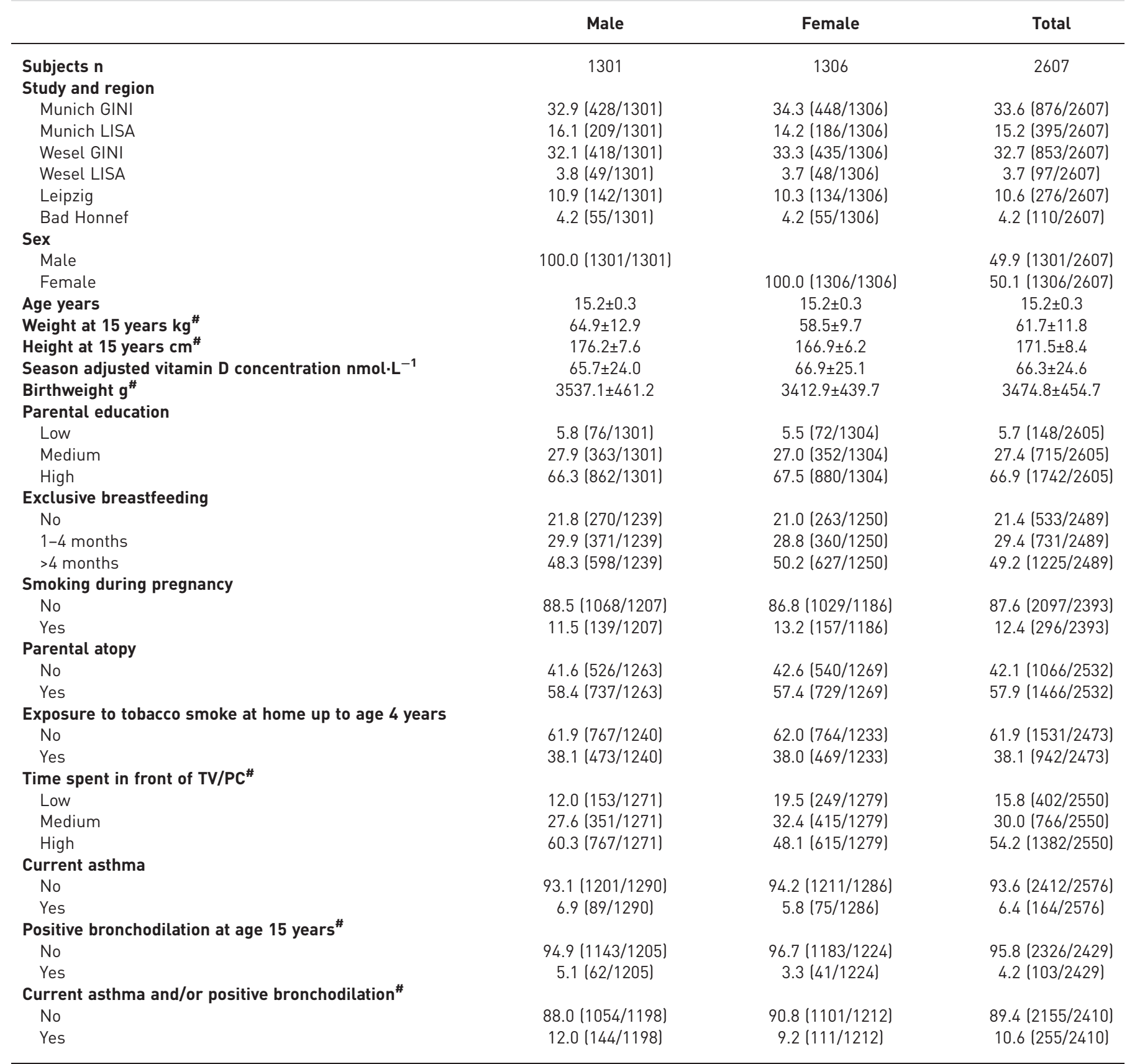

Data are presented as mean \pm SD or $\%(\mathrm{n} / \mathrm{N})$, unless otherwise stated. ${ }^{*}$ : significant difference between males and females (tested using chi-squared test or t-test).

remained statistically significant in the main model after adjustment for further confounding factors (FEV1: $\beta=0.010$, 95\% CI $0.002-0.017$; FVC: $\beta=0.020,95 \%$ CI $0.012-0.028)$. The ratio of FEV1 to FVC was negatively associated with $25(\mathrm{OH}) \mathrm{D}$; i.e. it decreased by $0.182 \%(\beta=-0.182,95 \% \mathrm{CI}-0.281$ to -0.084$)$ in the minimal model and $0.177 \%(\beta=-0.177,95 \%$ CI -0.286 to -0.067$)$ in the main model. Similar associations were observed when considering the $z$-scores calculated according to GLI (FEV1: $\beta=0.021,95 \%$ CI $0.005-0.037$; FVC: $\beta=0.039,95 \%$ CI $0.023-0.055$; FEV1/FVC: $\beta=-0.029,95 \%$ CI -0.046 to -0.011 in the main models). Flow rates (FEF25, FEF50, FEF75), mean flow rate (FEF25-75) and peak expiratory flow (PEF) were not significantly associated with $25(\mathrm{OH}) \mathrm{D}$ concentrations, neither in the minimal nor in the main model.

Table 4 presents the results for the associations between vitamin $\mathrm{D}$ and lung function measured postbronchodilation. As for the measurements pre-bronchodilation, FEV1 and FVC were positively significantly 
TABLE 2 Characteristics of spirometric lung function parameters measured pre- and post-bronchodilation, for study participants with valid vitamin $D$ measurements

\begin{tabular}{|c|c|c|c|}
\hline & Male & Female & Total \\
\hline Subjects $n$ & 1301 & 1306 & 2607 \\
\hline \multicolumn{4}{|l|}{ Before bronchodilation } \\
\hline FEV $1 L^{\#}$ & $3.82 \pm 0.64$ & $3.20 \pm 0.42$ & $3.50 \pm 0.62$ \\
\hline FVC L" & $4.51 \pm 0.76$ & $3.64 \pm 0.49$ & $4.07 \pm 0.77$ \\
\hline FEV $1 / F V C \%{ }^{\#}$ & $84.95 \pm 6.32$ & $88.12 \pm 6.10$ & $86.55 \pm 6.41$ \\
\hline PEF L. $S^{-1^{\#}}$ & $7.64 \pm 1.30$ & $6.48 \pm 0.97$ & $7.06 \pm 1.28$ \\
\hline FEF25 L. $\mathrm{s}^{-1^{\#}}$ & $6.52 \pm 1.26$ & $5.85 \pm 0.95$ & $6.18 \pm 1.16$ \\
\hline FEF50 L.S $\mathrm{S}^{-1^{\#}}$ & $4.64 \pm 1.11$ & $4.20 \pm 0.90$ & $4.42 \pm 1.03$ \\
\hline FEF75 L. $\mathrm{S}^{-1^{\#}}$ & $2.28 \pm 0.77$ & $2.12 \pm 0.64$ & $2.20 \pm 0.71$ \\
\hline FEF25-75 L. $\mathrm{S}^{-1^{\#}}$ & $4.07 \pm 0.99$ & $3.71 \pm 0.80$ & $3.89 \pm 0.91$ \\
\hline z-score FEV1 GLI & $-0.59 \pm 0.95$ & $-0.52 \pm 0.88$ & $-0.56 \pm 0.92$ \\
\hline z-score FVC GLI ${ }^{\#}$ & $-0.56 \pm 0.96$ & $-0.45 \pm 0.88$ & $-0.50 \pm 0.92$ \\
\hline z-score FEV1/FVC GLI & $-0.11 \pm 1.00$ & $-0.12 \pm 1.00$ & $-0.11 \pm 1.00$ \\
\hline z-score FEF75 GLI & $-0.14 \pm 0.94$ & $-0.11 \pm 0.92$ & $-0.13 \pm 0.93$ \\
\hline z-score $\mathrm{FEF}_{25-75} \mathrm{GLI}^{\#}$ & $-0.49 \pm 0.95$ & $-0.37 \pm 0.93$ & $-0.43 \pm 0.94$ \\
\hline \multicolumn{4}{|l|}{ After bronchodilation } \\
\hline FEV1 $L^{\#}$ & $3.94 \pm 0.65$ & $3.28 \pm 0.42$ & $3.61 \pm 0.64$ \\
\hline FVC L\# & $4.50 \pm 0.75$ & $3.63 \pm 0.49$ & $4.06 \pm 0.77$ \\
\hline $\mathrm{FEV}_{1} / \mathrm{FVC} \%{ }^{\#}$ & $87.71 \pm 5.58$ & $90.68 \pm 5.17$ & $89.20 \pm 5.58$ \\
\hline PEF L. $S^{-1^{\#}}$ & $7.78 \pm 1.31$ & $6.66 \pm 0.98$ & $7.22 \pm 1.28$ \\
\hline $\mathrm{FEF}_{25} \mathrm{~L} \cdot \mathrm{S}^{-1^{\#}}$ & $6.78 \pm 1.24$ & $6.08 \pm 0.96$ & $6.43 \pm 1.16$ \\
\hline FEF50 L.S $\mathrm{S}^{-1^{\#}}$ & $5.09 \pm 1.10$ & $4.60 \pm 0.87$ & $4.84 \pm 1.02$ \\
\hline FEF75 L. $\mathrm{S}^{-1^{\#}}$ & $2.64 \pm 0.85$ & $2.47 \pm 0.70$ & $2.55 \pm 0.78$ \\
\hline FEF25-75 L.s ${ }^{-1 \#}$ & $4.50 \pm 1.00$ & $4.11 \pm 0.78$ & $4.30 \pm 0.92$ \\
\hline z-score FEV1 GLI & $-0.34 \pm 0.95$ & $-0.30 \pm 0.88$ & $-0.32 \pm 0.92$ \\
\hline z-score FVC GLI ${ }^{\#}$ & $-0.56 \pm 0.96$ & $-0.46 \pm 0.90$ & $-0.51 \pm 0.93$ \\
\hline z-score FEV1/FVC GLI & $0.33 \pm 0.92$ & $0.31 \pm 0.91$ & $0.32 \pm 0.92$ \\
\hline z-score FEF75 GLI & $0.31 \pm 0.91$ & $0.38 \pm 0.91$ & $0.34 \pm 0.91$ \\
\hline z-score $\mathrm{FEF}_{25-75} \mathrm{GLI}^{\#}$ & $-0.05 \pm 0.89$ & $0.11 \pm 0.89$ & $0.03 \pm 0.89$ \\
\hline \multicolumn{4}{|c|}{$\begin{array}{l}\text { Data are presented as mean } \pm \text { SD. FEV1: forced expiratory volume in } 1 \mathrm{~s} \text {; FVC: forced vital capacity; } \\
\text { PEF: peak expiratory flow; FEF25, FEF50, FEF75: forced expiratory flow rates at } 25,50 \text { and } 75 \% \text { of exhaled } \\
\text { FVC; FEF25-75: mean flow rate between } 25 \text { and } 75 \% \text { of FVC; GLI: } z \text {-scores according to the Global Lung } \\
\text { function Initiative [23]. " : significant difference between males and females (tested using t-test). }\end{array}$} \\
\hline
\end{tabular}

related to 25(OH)D concentrations (main model: FEV1: $\beta=0.011,95 \%$ CI $0.004-0.018$; FVC: $\beta=0.019$, 95\% CI 0.011-0.027), whereas FEV1/FVC showed a negative relationship (main model: $\beta=-0.139,95 \%$ $\mathrm{CI}-0.236$ to -0.042 ). Comparable results were observed for the GLI $z$-scores.

Excluding asthmatics and those showing a positive bronchodilation response did not substantially affect the associations (figures 2 and 3 ).

\section{Discussion}

\section{Summary}

This cross-sectional study investigated the association between serum $25(\mathrm{OH}) \mathrm{D}$ concentrations and spirometric lung function indices measured before and after bronchodilation at 15 years of age. Serum 25 $(\mathrm{OH}) \mathrm{D}$ concentrations were positively associated with the volume-related lung function parameters $\mathrm{FEV} 1$ and FVC, and stronger effects were observed for FVC than FEV1. As the results were similar for lung function parameters measured pre- and post-bronchodilation, structural changes in peripheral airways are suggested. The findings did not substantially change if analyses were restricted to apparently lung healthy subjects, excluding those with positive bronchodilation response or asthma.

\section{Results from other studies}

Several previous cross-sectional studies have analysed the association between vitamin D and lung function, but only a few studies have focused on adolescents $[8,10,11,28]$. For instance, the study by YAO et al. [8], conducted in 1282 Taiwanese children and adolescents aged 5 to 18 years, observed positive and significant 
TABLE 3 Results for the association between season-adjusted 25-hydroxyvitamin D concentrations per $10 \mathrm{nmol} \cdot \mathrm{L}^{-1}$ increase and lung function measurements pre-bronchodilation

\begin{tabular}{|c|c|c|c|c|c|c|}
\hline & \multicolumn{3}{|c|}{ Model $1^{\#}$} & \multicolumn{3}{|c|}{ Model 2" } \\
\hline & $\boldsymbol{\beta}$ & $95 \% \mathrm{Cl}$ & p-value & $\boldsymbol{\beta}$ & $95 \% \mathrm{Cl}$ & p-value \\
\hline FEV1 & 0.013 & $0.007-0.020$ & $<0.001$ & 0.010 & $0.002-0.017$ & 0.010 \\
\hline FVC & 0.024 & $0.017-0.031$ & $<0.001$ & 0.020 & $0.012-0.028$ & $<0.001$ \\
\hline $\mathrm{FEV}_{1} / \mathrm{FVC}$ & -0.182 & $-0.281--0.084$ & $<0.001$ & -0.177 & $-0.286--0.067$ & 0.002 \\
\hline PEF & 0.013 & $-0.004-0.029$ & 0.125 & 0.014 & $-0.004-0.033$ & 0.135 \\
\hline FEF25 & -0.001 & $-0.018-0.016$ & 0.896 & 0.004 & $-0.015-0.023$ & 0.690 \\
\hline FEF50 & 0.000 & $-0.015-0.016$ & 0.975 & -0.003 & $-0.020-0.015$ & 0.773 \\
\hline FEF75 & -0.005 & $-0.015-0.006$ & 0.419 & -0.008 & $-0.021-0.004$ & 0.182 \\
\hline FEF25-75 & 0.000 & $-0.014-0.014$ & 0.993 & -0.004 & $-0.019-0.011$ & 0.619 \\
\hline z-score FEV1 GLI & 0.028 & $0.014-0.042$ & $<0.001$ & 0.021 & $0.005-0.037$ & 0.010 \\
\hline z-score FVC GLI & 0.046 & $0.032-0.060$ & $<0.001$ & 0.039 & $0.023-0.055$ & $<0.001$ \\
\hline z-score FEV1/FVC GLI & -0.029 & $-0.045--0.014$ & $<0.001$ & -0.029 & $-0.046--0.011$ & 0.002 \\
\hline z-score FEF75 GLI & -0.006 & $-0.021-0.009$ & 0.465 & -0.010 & $-0.027-0.007$ & 0.250 \\
\hline z-score FEF25-75 GLI & -0.001 & $-0.016-0.014$ & 0.914 & -0.004 & $-0.021-0.013$ & 0.619 \\
\hline
\end{tabular}

Values presented in bold are statistically significant at $p<0.05$. $F E V_{1}$ : forced expiratory volume in $1 \mathrm{~s}$; FVC: forced vital capacity; PEF: peak expiratory flow; FEF25, FEF50, FEF75: forced expiratory flow rates at 25, 50 and $75 \%$ of exhaled FVC; FEF25-75: mean flow rate between 25 and 75\% of FVC; GLI: $z$-scores according to the Global Lung function Initiative [23]. \#: adjusted for sex, study and study region, age, height and weight at lung function testing. ": adjusted for all variables in Model 1 plus birthweight, time spent in front of a TV/PC, current asthma and/or positive bronchodilation response, exposure to tobacco smoke at home up to age 4 years, parental education, parental atopy, maternal smoking during pregnancy and breastfeeding.

associations between serum 25(OH)D concentrations and FEV1 and FVC: per ng.mL ${ }^{-1}$ increase in serum $25(\mathrm{OH}) \mathrm{D}, \mathrm{FEV} 1$ increased by $2.4 \mathrm{~mL}$ and FVC by $3.6 \mathrm{~mL}$. As these effect sizes correspond to increases by $9.6 \mathrm{~mL}$ and $14.4 \mathrm{~mL}$ for FEV1 and FVC, respectively, per $10 \mathrm{nmol} \cdot \mathrm{L}^{-1}$ increase in serum $25(\mathrm{OH}) \mathrm{D}$, these

TABLE 4 Results for the association between season-adjusted 25-hydroxyvitamin D concentrations per $10 \mathrm{nmol} \cdot \mathrm{L}^{-1}$ increase and lung function measurements post-bronchodilation

\begin{tabular}{|c|c|c|c|c|c|c|}
\hline & \multicolumn{3}{|c|}{ Model 1\# } & \multicolumn{3}{|c|}{ Model 2" } \\
\hline & $\boldsymbol{\beta}$ & $95 \% \mathrm{Cl}$ & p-value & $\boldsymbol{\beta}$ & $95 \% \mathrm{Cl}$ & p-value \\
\hline FEV 1 & 0.014 & $0.007-0.020$ & $<0.001$ & 0.011 & $0.004-0.018$ & 0.004 \\
\hline FVC & 0.023 & $0.015-0.030$ & $<0.001$ & 0.019 & $0.011-0.027$ & $<0.001$ \\
\hline $\mathrm{FEV}_{1} / \mathrm{FVC}$ & -0.148 & $-0.235--0.061$ & 0.001 & -0.139 & $-0.236--0.042$ & 0.005 \\
\hline PEF & 0.016 & $-0.001-0.033$ & 0.060 & 0.016 & $-0.003-0.035$ & 0.091 \\
\hline $\mathrm{FEF}_{25}$ & 0.004 & $-0.013-0.021$ & 0.617 & 0.011 & $-0.008-0.030$ & 0.259 \\
\hline FEF50 & 0.007 & $-0.008-0.022$ & 0.380 & 0.005 & $-0.012-0.022$ & 0.578 \\
\hline FEF75 & -0.005 & $-0.017-0.007$ & 0.449 & -0.010 & $-0.023-0.004$ & 0.168 \\
\hline FEF25-75 & 0.002 & $-0.011-0.016$ & 0.723 & -0.001 & $-0.016-0.015$ & 0.934 \\
\hline z-score FEV1 GLI & 0.030 & $0.016-0.045$ & $<0.001$ & 0.025 & $0.008-0.041$ & 0.003 \\
\hline z-score FVC GLI & 0.044 & $0.030-0.058$ & $<0.001$ & 0.038 & $0.022-0.053$ & $<0.001$ \\
\hline$z$-score $\mathrm{FEV}_{1} / \mathrm{FVC} \mathrm{GLI}$ & -0.025 & $-0.040--0.010$ & 0.001 & -0.024 & $-0.041--0.007$ & 0.005 \\
\hline z-score FEF75 GLI & -0.006 & $-0.021-0.009$ & 0.462 & -0.011 & $-0.028-0.006$ & 0.199 \\
\hline z-score FEF25-75 GLI & 0.002 & $-0.012-0.017$ & 0.756 & 0.000 & $-0.017-0.016$ & 0.956 \\
\hline
\end{tabular}

Values presented in bold are statistically significant at $p<0.05$. FEV1: forced expiratory volume in $1 \mathrm{~s}$; FVC: forced vital capacity; PEF: peak expiratory flow; FEF25, FEF50, FEF75: forced expiratory flow rates at 25, 50 and $75 \%$ of exhaled FVC; FEF25-75: mean flow rate between 25 and 75\% of FVC; GLI: $z$-scores according to the Global Lung function Initiative [23]. \#: adjusted for sex, study and study region, age, height and weight at lung function testing. ": adjusted for all variables in Model 1 plus birthweight, time spent in front of a TV/PC, current asthma and/or positive bronchodilation response, exposure to tobacco smoke at home up to age 4 years, parental education, parental atopy, maternal smoking during pregnancy and breastfeeding. 

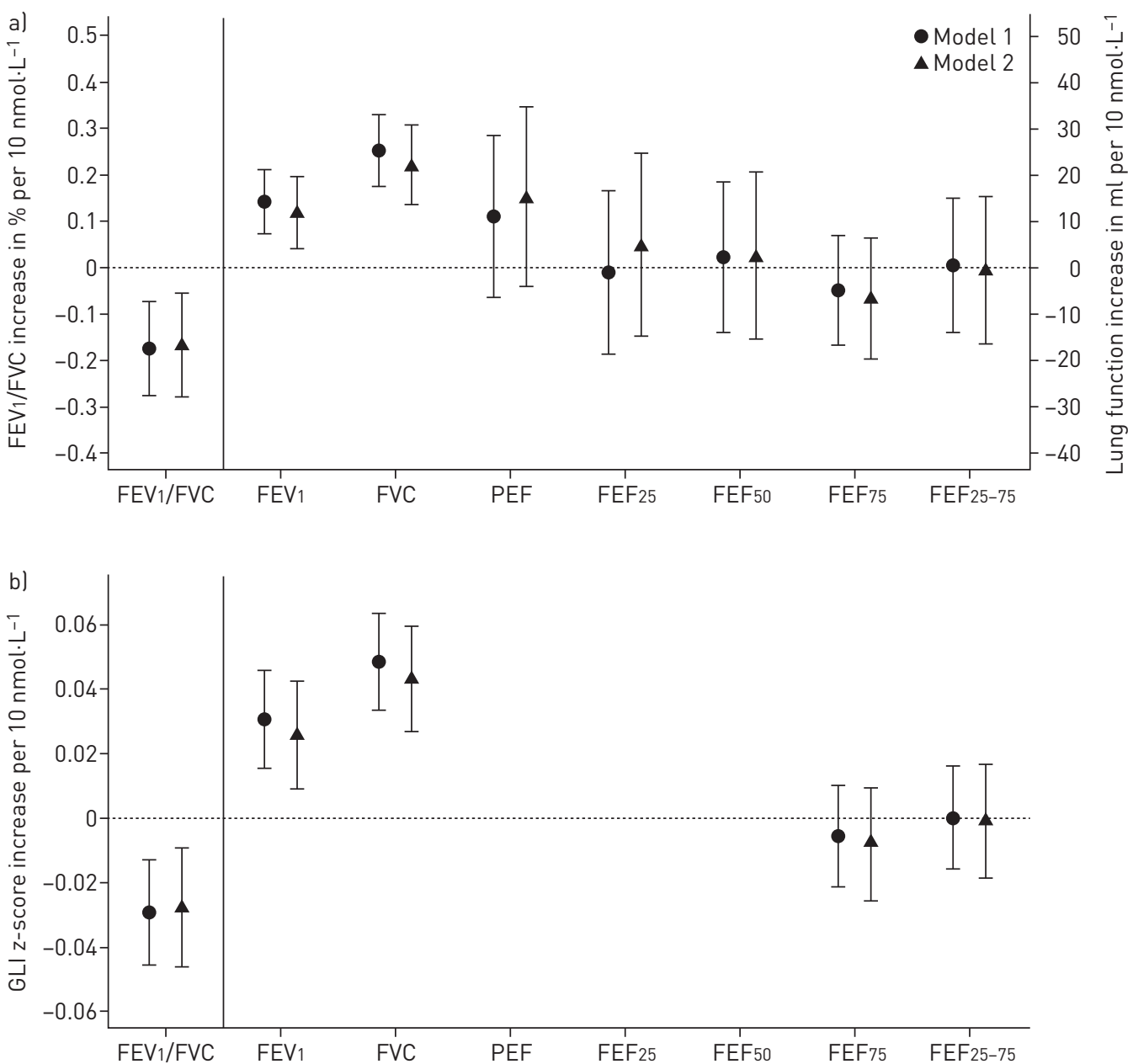

FIGURE 2 Results for the association between season-adjusted 25(OH)D concentrations per $10 \mathrm{nmol} \cdot \mathrm{L}^{-1}$ increase with a) spirometric lung function measurements and b) GLI z-scores pre-bronchodilation for the study population restricted to non-asthmatics and subjects with no positive bronchodilation response. Model 1: adjusted for sex, study and study region, age, height and weight at lung function testing. Model 2: adjusted for all variables in Model 1 plus birthweight, time spent in front of a TV/PC, exposure to tobacco smoke at home up to age 4 years, parental education, parental atopy, maternal smoking during pregnancy and breastfeeding. FEV1: forced expiratory volume in $1 \mathrm{~s}$; FVC: forced vital capacity; PEF: peak expiratory flow; FEF25, FEF50, FEF75: forced expiratory flow rates at 25, 50 and 75\% of exhaled FVC; FEF25-75: mean flow rate between 25 and 75\% of FVC; GLI: z-scores according to the Global Lung function Initiative.

effects are comparable to the results of the current study. Because, in the study by YAO et al. [8] as well as in the current study, stronger effects were observed for FVC than for FEV1, the associations with vitamin $\mathrm{D}$ might be indicative of changes in the lung volume rather than airway alterations with airflow limitations.

By contrast, in 3735 adolescents and adults aged 13 to 69 years participating in the Canadian Health Measures Survey [28], 25(OH)D concentrations were significantly negatively associated with FEV1/FVC, but no association was found with FEV1 and FVC. Tolppanen and co-workers $[10,11]$ investigated the same research question in two studies. In the first study, which was a cross-sectional study in adolescents aged 12-19 years and adults aged 20-59 years from the third National Health and Nutrition Examination Survey, a positive association between serum 25(OH)D concentrations and FVC was found in adolescents as well as in adults [10]. The relationship with FEV1 was significant among adults, but not among adolescents. However, the effect of serum $25(\mathrm{OH}) \mathrm{D}$ concentrations was stronger for FVC than for FEV1, irrespective of the significance of the results. In the second study, which was based on data from the population-based Avon Longitudinal Study of Parents and Children, spirometric lung function parameters measured after bronchodilation were taken into account [11]. In this prospective study, $25(\mathrm{OH}) \mathrm{D}$ concentrations were measured at the age of 10 years, whereas lung function testing was performed at the age of 15 years. Furthermore, a differentiation between $25(\mathrm{OH}) \mathrm{D}_{2}$ and $25(\mathrm{OH}) \mathrm{D}_{3}$ was included: $25(\mathrm{OH}) \mathrm{D}_{2}$ concentrations were weakly associated with FEV1 and FVC, whereas $25(\mathrm{OH}) \mathrm{D}_{3}$ concentrations were not 

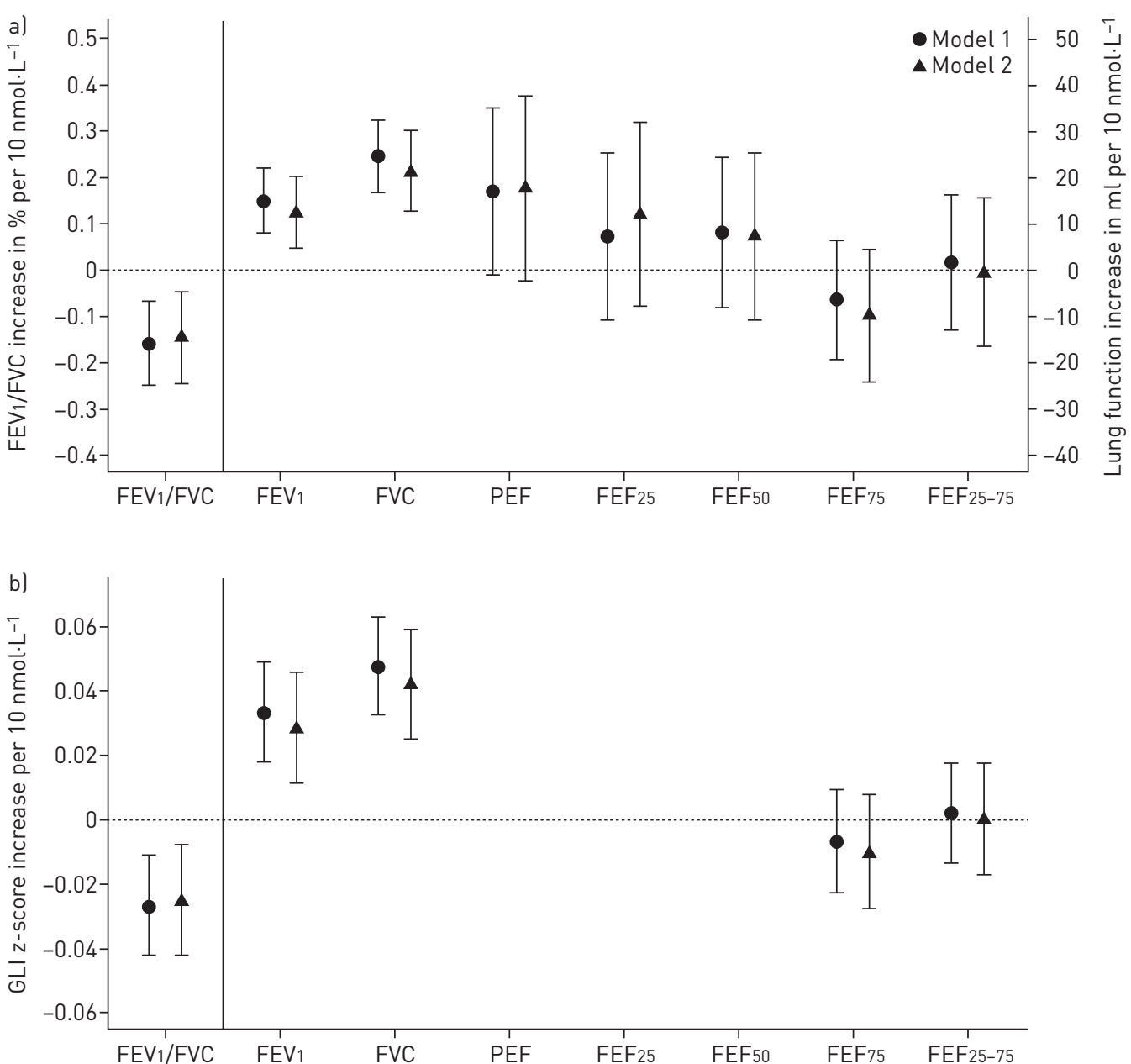

FIGURE 3 Results for the association between season-adjusted 25(OH)D concentrations per $10 \mathrm{nmol} \cdot \mathrm{L}^{-1}$ increase with a) spirometric lung function measurements and b) GLI z-scores post-bronchodilation for the study population restricted to non-asthmatics and subjects with no positive bronchodilation response. Model 1: adjusted for sex, study and study region, age, height and weight at lung function testing. Model 2: adjusted for all variables in Model 1 plus birthweight, time spent in front of a TV/PC, exposure to tobacco smoke at home up to age 4 years, parental education, parental atopy, maternal smoking during pregnancy and breastfeeding. FEV1: forced expiratory volume in $1 \mathrm{~s}$; FVC: forced vital capacity; PEF: peak expiratory flow; FEF25, FEF50, FEF75: forced expiratory flow rates at 25, 50 and 75\% of exhaled FVC; FEF25-75: mean flow rate between 25 and 75\% of FVC; GLI: z-scores according to the Global Lung function Initiative.

associated with lung function. However, none of these studies reported results for lung function parameters measured pre- as well as post-bronchodilation.

The different results of the mentioned studies might also be due to different confounding variables and different measurement methods [29] for the 25(OH)D concentrations. Only the study by YAO et al. [8] used the same assay to measure vitamin D concentrations as was used in the GINIplus and LISAplus studies. As the vitamin D concentrations were categorised in some studies, $25(\mathrm{OH}) \mathrm{D}$ measurements are not directly comparable between studies.

In summary, although there are differences in the studies regarding the association between vitamin $\mathrm{D}$ concentrations and lung function in adolescence, the findings are related to FVC, suggesting an involvement of peripheral lung structures.

\section{Biological mechanisms}

The exact mechanisms underlying the association between vitamin D and lung function are not completely understood. However, there are some hypothesised mechanisms that might explain this relationship. One possible explanation is the role vitamin D plays in airway remodelling [30]. Airway remodelling occurs early in asthmatics and is characterised by increased airway smooth muscle mass, fibrosis with alterations in extracellular matrix composition as well as subepithelial membrane thickening, all indicating structural 
changes [30, 31]. As the results of the current study have shown similar associations for lung function measurements pre- and post-bronchodilation, structural, and not functional, changes in the lung are suggested, which would support the hypothesis of vitamin D influencing airway remodelling. Another hypothesis might be related to the influence of vitamin $\mathrm{D}$ on the development and modulation of the immune system $[1,32,33]$. For instance, the active vitamin D metabolite can lead to an inhibition of the activation and proliferation of T-lymphocytes $[32,34]$. It has been shown that Th1-associated cytokine production is inhibited, whereas Th2 responses could be inhibited as well as enhanced [32]. Both Th1 and Th2 cells are involved in asthma by producing pro- and anti-inflammatory cytokines. Furthermore, vitamin $\mathrm{D}$ might support regulatory $\mathrm{T}$-cells, which inhibit airway hyperresponsiveness and airway inflammation [32]. Lung development, growth and maturation as well as surfactant secretion might also be affected by vitamin D via its receptors present in fetal type II alveolar cells [30, 32, 35]. The study by ZoskY et al. [36, 37] has shown that vitamin D-deficient mice have a significantly reduced lung volume, in terms of thoracic gas volume, compared to vitamin D-replete mice. Lower vitamin D concentrations also resulted in reduced numbers of alveoli in female mice. Furthermore, an influence of vitamin D on the alveolar epithelialmesenchymal interactions was observed in the study by SAKURAI et al. [37, 38]. The active vitamin D metabolite inhibits apoptosis and thus increases alveolar type II cell proliferation. Besides surfactant synthesis, the production of interstitial fibres, i.e. collagen type I alpha 1 , is affected by vitamin D deficiency [39]. These experimental studies support the hypothesis that vitamin D affects the alveolar region of the lung, causing changes in lung volume rather than airflow limitations, which is in line with our and other findings that FVC is mainly affected by serum $25(\mathrm{OH}) \mathrm{D}$ concentrations.

Besides these mechanisms, genetic variation and epigenetic mechanisms might also be involved in regulating the relationship between vitamin D concentrations and respiratory health $[40,41]$.

\section{Strengths and limitations}

One of the strengths of this study is the lung function measurement pre- and post-bronchodilation, as well as the inclusion of forced flow rates by spirometry. As lung function testing was also performed after inhalation of salbutamol, which has an airway-widening effect, this study allowed to investigate whether the association between vitamin D concentrations and lung function parameters is only based on reversible lung function impairment or whether it is rather based on fixed, structural changes of the lung. Furthermore, parameters of small airways and airway narrowing were also measured as well as the commonly used parameters indicating lung volume and size and airway obstruction. Another strength of this study is that the serum 25(OH)D concentrations were adjusted for the exact date of blood sampling to carefully correct for the seasonal variation of vitamin D. Most other studies only adjusted for season of sampling.

Besides these strengths, this study also has some limitations. As with most other studies, our findings are based on a cross-sectional study design. Thus, reverse causation cannot be ruled out, even though the biological mechanisms probably do not support the notion. Theoretically, it is possible that lung function affects vitamin D concentrations, i.e. adolescents having asthma and therefore a reduced lung function are less active and spend less time outdoors. However, sensitivity analyses restricted to non-asthmatics and those without a positive bronchodilation response were conducted and showed similar results. We therefore consider a reverse causation unlikely. Moreover, the standardised $z$-scores according to GLI did not fit very well to the current study population [42]. Thus, the regression models calculated for the association between serum 25(OH)D concentrations and GLI $z$-scores were also adjusted for sex as well as age and height at lung function measurement. It is thus unlikely that measurement error or insufficient standardisation errors for lung function data would introduce major bias. Another limitation of the current study might be the restricted generalisability of these findings to the general German population of adolescents, because only four regions with two metropolitan areas were used to recruit study subjects. In addition, participants of parents with a high socioeconomic status are overrepresented in the GINIplus and LISAplus studies. However, whether the participants were assigned to the intervention (with hydrolysed versus cow's milk formulae) or the observation arm of the GINIplus study did not affect the observed associations (data not shown). Another common problem in prospective birth cohorts is loss to follow-up.

\section{Conclusion}

The results of this study suggest that higher vitamin D concentrations are associated with higher lung volume-related spirometric parameters in adolescence. Similar associations were observed for lung function parameters measured pre- and post-bronchodilation, suggesting structural changes in peripheral airways.

\section{Acknowledgements}

We thank the families for their participation in the study, the obstetric units for allowing recruitment, and the GINI and LISA study teams for their excellent work. 


\section{Study groups}

\section{The GINIplus 15 Study Group}

Helmholtz Zentrum München, German Research Center for Environmental Health, Institute of Epidemiology I, Neuherberg ( J. Heinrich, I. Brüske, H. Schulz, M. Standl, M. Schnappinger, M. Sußmann, E. Thiering, C. Tiesler, C. Flexeder, C. Zeller); Marien-Hospital Wesel, Department of Pediatrics, Research Institute, Wesel (D. Berdel, A. von Berg, B. Filipiak-Pittroff); Ludwig-Maximilians-University, Dr. von Hauner Children's Hospital, Munich (S. Koletzko, K. Werkstetter); Technical University Munich, Department of Pediatrics, and Deutsche Rentenversicherung Bayern Süd (C.P. Bauer, U. Hoffmann); IUF Leibniz Research Institute for Environmental Medicine, Düsseldorf (B. Hoffmann, E. Link, C. Klümper, U. Krämer, D. Sugiri)

The LISAplus 15 Study Group

Helmholtz Zentrum München, German Research Center for Environmental Health, Institute of Epidemiology I, Neuherberg ( J. Heinrich, I. Brüske, H. Schulz, M. Standl, M. Schnappinger, M. Sußmann, E. Thiering, C. Tiesler, C. Flexeder, C. Zeller); Marien-Hospital Wesel, Department of Pediatrics, Research Institute, Wesel (A. von Berg); Pediatric Practice, Bad Honnef (B. Schaaf); Technical University Munich, Department of Pediatrics, Munich (U. Hoffmann); Helmholtz Centre for Environmental Research - UFZ, Department of Environmental Immunology/Core Facility Studies, Leipzig (I. Lehmann, M. Bauer, G. Herberth, J. Müller, S. Röder, M. Schilde); Municipal Hospital 'St Georg', Department of Pediatrics, Leipzig (M. Borte, U. Diez, C. Dorn, E. Braun); Technical University Munich, ZAUM - Center for Allergy and Environment, Munich (M. Ollert, J. Grosch).

\section{References}

1 Bozzetto S, Carraro S, Giordano G, et al. Asthma, allergy and respiratory infections: the vitamin D hypothesis. Allergy 2012; 67: 10-17.

2 Holick MF. Vitamin D deficiency. $N$ Engl J Med 2007; 357: 266-281.

3 Zerwekh JE. Blood biomarkers of vitamin D status. Am J Clin Nutr 2008; 87: 1087S-1091S.

4 Della Giustina A, Landi M, Bellini F, et al. Vitamin D, allergies and asthma: focus on pediatric patients. World Allergy Organ J 2014; 7: 27.

5 Wawro N, Heinrich J, Thiering E, et al. Serum 25(OH)D concentrations and atopic diseases at age 10: results from the GINIplus and LISAplus birth cohort studies. BMC Pediatr 2014; 14: 286.

$6 \quad$ Wjst M. The vitamin D slant on allergy. Pediatr Allergy Immunol 2006; 17: 477-483.

7 Damera G, Fogle HW, Lim P, et al. Vitamin D inhibits growth of human airway smooth muscle cells through growth factor-induced phosphorylation of retinoblastoma protein and checkpoint kinase 1. Br J Pharmacol 2009; 158: $1429-1441$.

8 Yao TC, Tu YL, Chang SW, et al. Serum 25-hydroxyvitamin D levels in relation to lung function and exhaled nitric oxide in children. J Pediatr 2014; 165: 1098-1103.e1.

9 Cremers E, Thijs C, Penders J, et al. Maternal and child's vitamin D supplement use and vitamin D level in relation to childhood lung function: the KOALA Birth Cohort Study. Thorax 2011; 66: 474-480.

10 Tolppanen AM, Williams D, Henderson J, et al. Serum 25-hydroxy-vitamin D and ionised calcium in relation to lung function and allergen skin tests. Eur J Clin Nutr 2011; 65: 493-500.

11 Tolppanen AM, Sayers A, Granell R, et al. Prospective association of 25-hydroxyvitamin D3 and D2 with childhood lung function, asthma, wheezing, and flexural dermatitis. Epidemiology 2013; 24: 310-319.

12 Black PN, Scragg R. Relationship between serum 25-hydroxyvitamin D and pulmonary function in the Third National Health and Nutrition Examination Survey. Chest 2005; 128: 3792-3798.

13 Larose TL, Langhammer A, Chen Y, et al. Serum 25-hydroxyvitamin D levels and lung function in adults with asthma: the HUNT Study. Eur Respir J 2015; 45: 1019-1026.

14 Thuesen BH, Skaaby T, Husemoen LL, et al. The association of serum 25-OH vitamin D with atopy, asthma, and lung function in a prospective study of Danish adults. Clin Exp Allergy 2015; 45: 265-272.

15 Thuesen BH, Heede NG, Tang L, et al. No association between vitamin D and atopy, asthma, lung function or atopic dermatitis: a prospective study in adults. Allergy 2015; 70: 1501-1504.

16 Brumpton BM, Langhammer A, Henriksen AH, et al. Vitamin D and lung function decline in adults with asthma: the HUNT Study. Am J Epidemiol 2016; 183: 739-746.

17 von Berg A, Koletzko S, Grübl A, et al. The effect of hydrolyzed cow's milk formula for allergy prevention in the first year of life: the German Infant Nutritional Intervention Study, a randomized double-blind trial. J Allergy Clin Immunol 2003; 111: 533-540.

18 von Berg A, Krämer U, Link E, et al. Impact of early feeding on childhood eczema: development after nutritional intervention compared with the natural course - the GINIplus study up to the age of 6 years. Clin Exp Allergy 2010; 40: 627-636

19 Heinrich J, Bolte G, Hölscher B, et al. Allergens and endotoxin on mothers' mattresses and total immunoglobulin E in cord blood of neonates. Eur Respir J 2002; 20: 617-623.

20 Zutavern A, Rzehak P, Brockow I, et al. Day care in relation to respiratory-tract and gastrointestinal infections in a German birth cohort study. Acta Paediatr 2007; 96: 1494-1499.

21 Miller MR, Hankinson J, Brusasco V, et al. Standardisation of spirometry. Eur Respir J 2005; 26: 319-338

22 Pellegrino R, Viegi G, Brusasco V, et al. Interpretative strategies for lung function tests. Eur Respir J 2005; 26 : 948-968.

23 Quanjer PH, Stanojevic S, Cole TJ, et al. Multi-ethnic reference values for spirometry for the 3-95-yr age range: the global lung function 2012 equations. Eur Respir J 2012; 40: 1324-1343.

24 R Core Team. R: A Language and Environment for Statistical Computing. Vienna, The R Foundation, 2015. Available from: www.R-project.org 
28 Niruban SJ, Alagiakrishnan K, Beach J, et al. Association between vitamin D and respiratory outcomes in Canadian adolescents and adults. J Asthma 2015; 52: 653-661.

29 Freeman J, Wilson K, Spears R, et al. Performance evaluation of four 25-hydroxyvitamin D assays to measure 25-hydroxyvitamin D2. Clin Biochem 2015; 48: 1097-1104.

30 Berraies A, Hamzaoui K, Hamzaoui A. Link between vitamin D and airway remodeling. J Asthma Allergy 2014; 7: 23-30.

31 Mann EH, Chambers ES, Pfeffer PE, et al. Immunoregulatory mechanisms of vitamin D relevant to respiratory health and asthma. Ann NY Acad Sci 2014; 1317: 57-69.

32 Lange NE, Litonjua A, Hawrylowicz CM, et al. Vitamin D, the immune system and asthma. Expert Rev Clin Immunol 2009; 5: 693-702.

33 Muehleisen B, Gallo RL. Vitamin D in allergic disease: shedding light on a complex problem. J Allergy Clin Immunol 2013; 131: 324-329.

34 van Etten E, Stoffels K, Gysemans C, et al. Regulation of vitamin D homeostasis: implications for the immune system. Nutr Rev 2008; 66: Suppl. 2, S125-S134.

35 Mirzakhani H, Al-Garawi A, Weiss ST, et al. Vitamin D and the development of allergic disease: how important is it? Clin Exp Allergy 2015; 45: 114-125.

36 Zosky GR, Berry LJ, Elliot JG, et al. Vitamin D deficiency causes deficits in lung function and alters lung structure. Am J Respir Crit Care Med 2011; 183: 1336-1343.

37 Lykkedegn S, Sorensen GL, Beck-Nielsen SS, et al. The impact of vitamin D on fetal and neonatal lung maturation. A systematic review. Am J Physiol Lung Cell Mol Physiol 2015; 308: L587-L602.

38 Sakurai R, Shin E, Fonseca S, et al. $1 \alpha, 25(\mathrm{OH})_{2} \mathrm{D}_{3}$ and its 3-epimer promote rat lung alveolar epithelial-mesenchymal interactions and inhibit lipofibroblast apoptosis. Am J Physiol Lung Cell Mol Physiol 2009; 297: L496-L505.

39 Chen L, Wilson R, Bennett E, et al. Identification of vitamin D sensitive pathways during lung development. Respir Res 2016; 17: 47.

40 Junge KM, Bauer T, Geissler S, et al. Increased vitamin D levels at birth and in early infancy increase offspring allergy risk - evidence for involvement of epigenetic mechanisms. J Allergy Clin Immunol 2016; 137: 610-613.

41 Hansen JG, Gao W, Dupuis J, et al. Association of 25-hydroxyvitamin D status and genetic variation in the vitamin D metabolic pathway with FEV1 in the Framingham Heart Study. Respir Res 2015; 16: 81.

42 Hüls A, Krämer U, Gappa M, et al. Age dependency of GLI reference values compared with paediatric lung function data in two German studies (GINIplus and LUNOKID). PLoS One 2016; 11: e0159678. 